\title{
Which treatment protocol is better in rehabilitation of joint contracture?
}

\author{
(1) Duygu Tecer ${ }^{1}$, (1) Evren Yaşar $^{2}$, (1) Emre Adıgüzel ${ }^{3}$, (1) Serdar Kesikburun², (1) Özlem Köroğlu², \\ (1) M. Ali Taşkaynatan ${ }^{4}$, (1) Ahmet Özgül ${ }^{5}$, (1) A. Kenan Tan²
}

1 University of Health Sciences Turkey, Gülhane Training and Research Hospital, Clinic of Rheumatology, Ankara, Turkey

2University of Health Sciences Turkey, Gülhane Faculty of Medicine, Department of Physical Medicine and Rehabilitation, Ankara, Turkey

${ }^{3}$ Gaziler Training and Research Hospital, Clinic of Physical Medicine and Rehabilitation Ankara, Turkey

${ }^{4}$ Memorial Hospital, Clinic of Physical Medicine and Rehabilitation, Istanbul, Turkey

${ }^{5}$ Near East University, University of Kyrenia Faculty of Medicine, Department of Physical Medicine and Rehabilitation, Mersin, Turkey

Date submitted:

3.06.2019

Date accepted:

7.11.2019

Online publication date:

15.03.2020

\section{Corresponding Author:}

Duygu Tecer MD, University of Health Sciences Turkey, Gülhane Training and Research Hospital, Clinic of Rheumatology, Ankara, Turkey duygu-tecer@hotmail.com

ORCID:

orcid.org/0000-0002-8816-6181

Keywords: Joint contracture, rehabilitation, physical therapy

\begin{abstract}
Aim: To investigate demographic features, treatment response and factors affecting improvement in patients with joint contracture.

Methods: Three hundred sixty-one in patients with decreased range of motion were included in this retrospectively observational study. The demographic and clinical characteristics of patients were recorded. According to the physical therapy modalities, patients were divided into three subgroups. All patients received conventional therapy, which consisted of hot pack, therapeutic ultrasound, and stretching exercises. In addition to the conventional therapy, transcutaneous electrical nerve stimulation (TENS) was applied to the patients in group $A$, hydrotherapy (whirlpool) to the patients in group B, TENS and hydrotherapy to the patients in group C.
\end{abstract}

Results: There were 130 (36\%) knee, 66 (18.3\%) ankle, 58 (16.1\%) elbow, 49 (13.6\%) wrist, 40 $(11.1 \%)$ shoulder, and 18 (5\%) hip joint cases. Patients had statistically significant improvements of all decreased joint range of motion $(p<0.001)$. Therapy of more than 30 sessions improved only knee flexion and wrist extension significantly. A negative correlation was detected between age and the difference in wrist extension $(r=-0.324, p=0.023)$. Improvements of knee flexion and elbow flexion were significantly better in the acute group than in subacute and chronic groups $(p=0.03, p=0.036)$. There was no statistically significant difference in range of motion of the elbow between the patients who used and those who did not use an adjustable elbow contracture orthosis. There was no statistically significant difference between the treatment protocols.

Conclusion: Therapy of more than 30 sessions is useful for only knee flexion and wrist extension contracture. Adding TENS or hydrotherapy have no additional benefit on conventional treatment.

\section{Introduction}

Contracture is the molecular shortening of connective tissue. Joint contracture is characterized by limited range of motion secondary to periarticular connective tissue changes (1). Multiple factors are associated with posttraumatic joint contracture most importantly occurring with pain, prolonged joint positioning (immobility), adhesions, heterotopic bone formation, joint incongruity and periarticular connective tissue changes (2). Upper limb joint contractures may lead to loss of ability to dress or to eat independently. Lower limb contractures may lead to imbalance and the inability to walk unaided with the consequent higher risk of being housebound or confined to bed (3). Also, joint contractures increase the risk of pain, pressure ulcers and fall. The main aim of improving range of motion is to reduce 
impairments, to increase function for the daily life activities and work and leisure activities and to prevent long term disability $(1,3)$. Therapeutic considerations in the treatment of joint contractures include safety, efficacy, availability, cost, and time. Therapy modalities for the management of joint contracture include passive stretching, splinting, application of serial plasters, joint mobilization, injection of botulinum toxin, electrical stimulation, and surgical manipulations $(1,4)$.

Joint contracture is a common problem in physical medicine and rehabilitation or orthopedic practices. However, there have been few studies which have investigated the management and long-term follow-up results of orthopedic joint contracture rehabilitation (5-7). In this retrospective study, the data on inpatients with joint contracture are presented and it was aimed to define the factors which affected rehabilitation outcomes. This study enlightens optimal therapy methods, therapy time, therapy period and other factors that can affect the outcomes in patients with joint contracture.

\section{Methods}

Approval of the Local Research Ethics Committee of our tertiary hospital was obtained before initiating the study (Gülhane Training and Research Hospital, January 02, 2013, 12th session). The research protocol complies with the Declaration of Helsinki and written informed consent was obtained from all participants.

In our study, we retrospectively evaluated the medical records of inpatients with decreased range of motion who were treated between January 2009 and January 2013. Patients with any central or peripheral nervous system disorder, primary muscle disease, congenital malformation, systemic arthritis, systemic or metabolic disease or burn were excluded.

This study was designed as a retrospective observational study in our national tertiary rehabilitation center. The medical records of the inpatients were obtained from the electronic database of our hospital. The demographic and clinical characteristics including the patient's age, gender, etiology of joint contracture, duration of disease, presence of fractures and fixator, range of motion before and after treatment, number of physical therapy sessions, use of orthotics, and type of physical therapy were evaluated. Passive range of motion was measured with a goniometer by the neutral zero method. Comparison between subgroups was performed. Time to the initiation of treatment was classified into three subgroups as follows; acute period (first 3 months of disease onset), subacute period (3-6 months) and chronic period (>6 months). According to the number of therapy sessions, patients were divided into two subgroups as follows; group 1 (up to 30 sessions) and group 2 (more than 30 sessions). Also, according to the physical therapy modalities, patients were divided into three subgroups. All patients received conventional therapy, which consisted of hot pack, therapeutic ultrasound, stretching exercises. In addition to the conventional therapy, transcutaneous electrical nerve stimulation (TENS) was applied to the patients in group A, hydrotherapy (whirlpool) to the patients in group $B$, and TENS and hydrotherapy to the patients in group C. Physiotherapists applied stretching exercises manually for 20 minutes per therapy session. The contracted joint was stretched until the patient felt discomfort, but not pain. Stretching was sustained for at least 20 seconds. TENS was administered for 20 minutes in each therapy session. The amplitude was increased until the patient felt a comfortable tingling sensation without motor contraction. Conventional TENS mode was used at high frequency and low intensity. The effects of the therapy session number and the physical therapy modalities on the rehabilitation outcomes were evaluated. An assessment was also made on the benefit of contracture orthosis in the management of elbow contracture.

\section{Statistical Analysis}

Statistical Package for Social Science (SPSS) version of 16.0 was used for analysis. (SPSS, Inc., Chicago, IL). The variables were investigated using visual (histograms, probability plots) and analytical methods (Kolmogorov-Smirnov, ShapiroWilk's test) to determine whether or not they were normally distributed. The Wilcoxon test was used to compare range of motion measurements before and after treatment. The MannWhitney $U$ test was applied in the comparison of differences in range of motion according to therapy sessions. The KruskalWallis test was used to compare measurements according to therapy groups and duration of the disease. The Mann-Whitney $U$ test was performed to test the significance of pairwise differences using Bonferroni correction to adjust for multiple comparisons. While investigating the associations between nonnormally distributed variables, the correlation coefficients and their significance were calculated using the Spearman test. A value of $p<0.05$ was considered statistically significant.

\section{Results}

Medical records of 423 inpatients with joint contracture were assessed for inclusion. Sixty-two patients were excluded due to concomitant peripheral nerve injury $(n=24)$, incomplete medical records $(n=13)$, traumatic brain injury $(n=11)$, burn $(n=9)$, and primary muscle disease $(n=5)$. A total of 361 inpatients (10 female, 351 male) who were treated for joint contracture related to orthopedic conditions were included in this study. There were $130(36 \%)$ knee, 66 (18.3\%) ankle, 58 (16.1\%) elbow, 49 (13.6 $\%)$ wrist, 40 (11.1\%) shoulder, and $18(5 \%)$ hip joint cases. The mean age was $27.3 \pm 8.0$ years. Most of the contractures were due to fall $(39.6 \%)$. Other etiologies of joint contracture were as follows; strain $80(22.1 \%)$, motor vehicle collision (inside vehicle) $45(12.5 \%)$, gunshot wound $40(11.1 \%)$, motor vehicle collision (outside vehicle) $26(7.2 \%)$, crush injury $13(3.6 \%)$, knife wound $10(2.8 \%)$, malignancy $3(0.8 \%)$, infection $1(0.3 \%)$. The median 
therapy period was 49.0 (minimum: 7.0; maximum: 300.0 ) days 115 patients $(31.9 \%)$ received up to 30 sessions therapy and 246 patients $(68.1 \%)$ received more than 30 sessions.

According to range of motion before and after treatment, statistically significant improvements were determined in all joints with decreased range of motion (except hip internal and external rotation, Wilcoxon test) (Table 1). In terms of the number of sessions, therapy of more than 30 sessions resulted in significant improvements only in knee flexion and wrist extension ( $p<0.001, p=0.016$ respectively, Mann-Whitney $U$ test) (Table 2). Correlations between age and joint range of motion improvements were also assessed and a negative correlation was detected only between age and wrist extension ( $r=-0.324$, $p=0.023$, Spearman test) (Table 3 ). No statistically significant difference was determined in range of motion between the groups in respect of the fracture and/or fixator ( $p>0.05$, MannWhitney $U$ test). Improvements in knee flexion and elbow flexion in patients who initiated therapy in acute period were significantly better than in other groups $(p=0.03, p=0.036$ respectively, Kruskal-Wallis test).

Twenty-three of 58 patients with elbow contracture used contracture orthosis. The median change in elbow flexion was 15 degree (minimum: 0, maximum: 60 ) in the patient group who used an adjustable elbow contracture orthosis and 10 degree (minimum: 0, maximum: 55) in the patient group who did not use. The median change in elbow extension was 10 degree (minimum: -10, maximum: 40) in the patient group who used contracture orthosis and 5 degree (minimum: 0, maximum: 60) in the patient group who did not use. Although changes in elbow flexion and extension degree tended to be higher in patients who used an adjustable elbow contracture orthosis than who did not use, this difference was not statistically significant $(p>0.05$, Mann-Whitney $U$ test). The median changes in elbow pronation and supination degree were 10 degree, there was no statistically significant difference ( $p>0.05$, Mann-Whitney $U$ test).

According to the therapy groups, 109 (30.2\%) patients were in group A, $138(38.2 \%)$ patients were in group B, and 114 $(31.6 \%)$ were in group $C$. There was no statistically significant difference among the treatment protocols of group $A, B$ and $C$ (all p>0.05, Kruskal Wallis test) (Table 4).

\section{Discussion}

This study on 361 inpatients investigated the long-term results of joint contracture rehabilitation. Statistically significant improvement was achieved in all joints and more than 30 treatment sessions provided beneficial effects that increased knee flexion and wrist extension. It has been previously suggested that the knee and the elbow joints are more prone to contracture, especially after injury or surgery (8). Concordant with these data, in our study, the joint most affected by

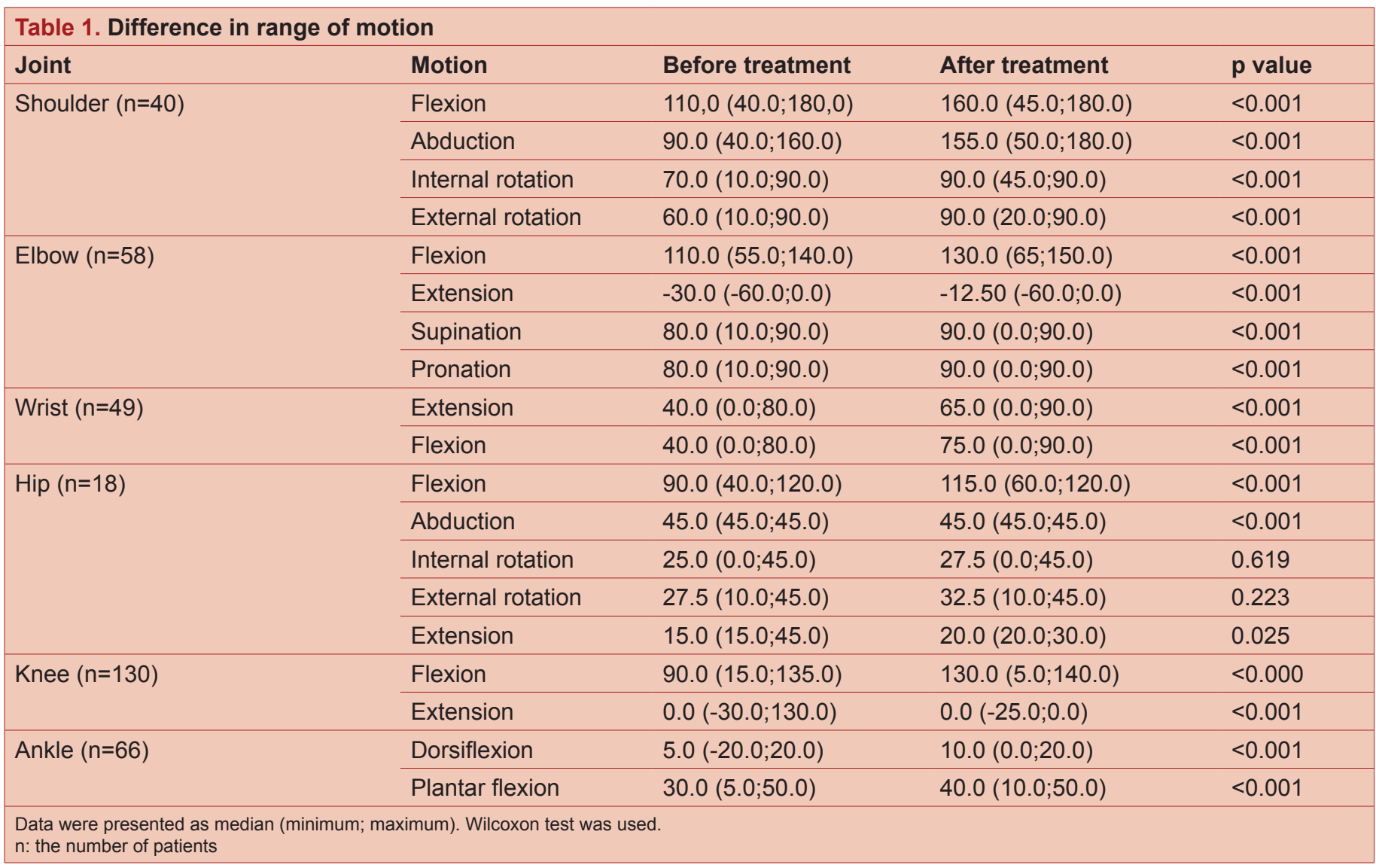


contracture in the lower extremities was the knee and that in the upper extremities was the elbow.

In daily practice, joint contracture, which is defined as a limitation of range of motion, is one of the most frequently encountered disorders in clinics of orthopedic and physical medicine and rehabilitation. Posttraumatic joint contractures, which are secondary to changes in joint structures, can be classified as intrinsic or extrinsic contractures. Knowledge of the affected structure in the joint contracture is essential for good management of contractures (9). Joint contracture is a sequel of trauma and it results from a combination of injury-related, diagnostic-related and therapy-related factors. Rehabilitation programs have an important role in obtaining the optimal range of motion, attaining independence in daily living activities and back to work (10).

In the current study, no statistically significant difference was found between the groups in respect of the treatment protocols. Therefore, the results of the current study suggest that conventional therapy is the most important part of the treatment.
In a systematic review by Harvey et al. (7), it is reported that conventional therapy (stretching) provides an increase in joint range of motion. In the current study, although TENS and hydrotherapy results showed no statistical significance, there were improvements in these treatment groups but level of improvement did not reach a significant level. Future prospective studies may be helpful to assess the exact benefit of these traditional therapy methods.

Stretch is widely used for the treatment and prevention of contractures. Stretch can be administered with splints or serial casting. Also, stretch can be self-administered or applied manually by therapists. In addition to conventional physical therapy for contractures, dynamic and static splinting modalities are frequently applied (11-14). Furia et al. (6) reviewed the effects of dynamic splinting and reported that dynamic splinting was associated with improvements in joint range of motion. The most used dynamic splint among the participants of this study was elbow contracture orthosis. These patients gained more elbow flexion but the difference from the patients

\begin{tabular}{|c|c|c|c|c|}
\hline Joint & Motion & $\leq 30$ session & $>30$ session & $p$ value \\
\hline \multirow[t]{5}{*}{ Shoulder } & & $\mathrm{n}=17$ & $n=23$ & \\
\hline & Flexion & $40.0(0.0 ; 100.0)$ & $50.0(0.0 ; 100.0)$ & $>0.05$ \\
\hline & Abduction & $40.0(5.0 ; 120.0)$ & $50.0(0.0 ; 130.0)$ & $>0.05$ \\
\hline & Internal rotation & $10.0(0.0 ; 80.0)$ & $15.0(0.0 ; 60.0)$ & $>0.05$ \\
\hline & External rotation & $10.0(0.0 ; 80.0)$ & $10.0(0.0 ; 60.0)$ & $>0.05$ \\
\hline \multirow[t]{5}{*}{ Elbow } & & $n=6$ & $n=42$ & \\
\hline & Flexion & $5.0(0.0 ; 40.0)$ & $10.0(0.0 ; 60.0)$ & $>0.05$ \\
\hline & Extension & $5.0(0.0 ; 10.0)$ & $10.0(-10.0 ; 60.0)$ & $>0.05$ \\
\hline & Supination & $7.5(0.0 ; 20.0)$ & $10.0(-90.0 ; 60.0)$ & $>0.05$ \\
\hline & Pronation & $5.0(0.0 ; 80.0)$ & $10.0(-90.0 ; 60.0)$ & $>0.05$ \\
\hline \multirow[t]{3}{*}{ Wrist } & & $n=17$ & $n=32$ & \\
\hline & Extension & $10.0(0.0 ; 50.0)$ & $20.0(0.0 ; 90.0)$ & 0.016 \\
\hline & Flexion & $20.0(0.0 ; 75.0)$ & $15.0(-5.0 ; 70.0)$ & $>0.05$ \\
\hline \multirow[t]{6}{*}{ Hip } & & $n=5$ & $n=13$ & \\
\hline & Flexion & $12.5(0.0 ; 65.0)$ & $20.0(0.0 ; 60.0)$ & $>0.05$ \\
\hline & Abduction & $5.0(0.0 ; 10.0)$ & $5.0(0.0 ; 10.0)$ & $>0.05$ \\
\hline & Internal rotation & $7.5(0.0 ; 20.0)$ & $0.0(-30.0 ; 5.0)$ & $>0.05$ \\
\hline & External rotation & $7.5(0.0 ; 10.0)$ & $0.0(-25.0 ; 10.0)$ & $>0.05$ \\
\hline & Extension & $5.0(5.0 ; 5.0)$ & $5.0(0.0 ; 5.0)$ & $>0.05$ \\
\hline \multirow[t]{3}{*}{ Knee } & & $\mathrm{n}=40$ & $\mathrm{n}=90$ & \\
\hline & Flexion & $20.0(-20.0 ; 60.0)$ & $40.0(-125.0 ; 115.0)$ & $<0.001$ \\
\hline & Extension & $1.0(0.0 ; 10.0)$ & $1.1(-130.0 ; 25.0)$ & $>0.05$ \\
\hline \multirow[t]{3}{*}{ Ankle } & & $\mathrm{n}=\mathbf{2 0}$ & $n=46$ & \\
\hline & Dorsiflexion & $5.0(0.0 ; 40.0)$ & $5.0(-20.0 ; 20.0)$ & $>0.05$ \\
\hline & Plantar flexion & $10.0(0.0 ; 40.0)$ & $10.0(-25.0 ; 40.0)$ & $>0.05$ \\
\hline
\end{tabular}


without contracture orthosis was not statistically significant. In the literature, there are studies that use different splinting protocols $(1,15)$. In the literature, at least 30-60 min of splint use was recommended and we used splinting for at least 2 hours (16). Some positive outcomes, including increased range of motion, improved patient satisfaction and reduced use of pain medication, were shown by some researchers (17-20). No significant difference in improvement in motion between static progressive and dynamic splinting protocols was reported by another prospective study (21). According to a recently published review, authors concluded that stretch did not have clinically important effects on joint mobility in people with or without neurological conditions if performed for less than seven months. In addition, stretch did not have clinically important short-term effects on the quality of life or pain in people with non-neurological conditions (22). Similarly, the later meta-analyses of randomised trials by the same authors showed that stretch did not have clinically important effects on joint mobility (23).

A negative correlation was determined between age and increasing range of motion in wrist extension in this study. This result seems to be expected because younger patients are prone to show better results due to elastic properties (24). But among all measured parameters, this result was present in only one joint and therefore this correlation should be carefully taken into consideration.
In the literature, there are emerging data that recommend early diagnosis and treatment of contractures (25). Similarly, according to the current results, early initiation of treatment was seen to be an important factor in achieving better rehabilitation outcomes. The improvements in knee and elbow flexion were remarkable particularly in patients who started therapy in acute period of the disease.

The major limitation of this study is the retrospective design. The number of treatment groups was not distributed equally. Also, there were many different etiologies, all of which could influence outcome. Lack of data about duration of contracture was another limitation of the study. However, to the best of our knowledge, there has been no previous study, which has included all joints with a long therapy duration and long follow-up period. Therefore, the results of this study may be of importance in guiding physical rehabilitation teams.

\section{Conclusion}

In conclusion, joint contracture or decreased range of motion is one of the most frequently seen problems in physical medicine and rehabilitation practice. Also, it is one of the most frequent orthopedic problems to complicate back to work generally. However, the literature about this period has been very poor. Therapy of more than 30 sessions is useful for only knee flexion and wrist extension contracture. Adding TENS or hydrotherapy has no additional benefit on conventional treatment.

\begin{tabular}{|c|c|c|c|}
\hline Joint & Motion & $r$ & p \\
\hline \multirow{4}{*}{ Shoulder $(n=40)$} & Flexion & 0.095 & $>0.05$ \\
\hline & Abduction & 0.190 & $>0.05$ \\
\hline & Internal rotation & 0.267 & $>0.05$ \\
\hline & External rotation & 0.167 & $>0.05$ \\
\hline \multirow[t]{4}{*}{ Elbow $(n=58)$} & Flexion & -0.168 & $>0.05$ \\
\hline & Extension & 0.246 & $>0.05$ \\
\hline & Supination & -0.251 & $>0.05$ \\
\hline & Pronation & -0.174 & $>0.05$ \\
\hline \multirow[t]{2}{*}{ Wrist $(n=49)$} & Extension & -0.314 & 0.023 \\
\hline & Flexion & -0.090 & $>0.05$ \\
\hline \multirow[t]{4}{*}{ Hip (n=18) } & Flexion & 0.300 & $>0.05$ \\
\hline & Internal rotation & 0.199 & $>0.05$ \\
\hline & External rotation & -0.410 & $>0.05$ \\
\hline & Extension & 0.118 & $>0.05$ \\
\hline \multirow[t]{2}{*}{ Knee $(n=130)$} & Flexion & -0.045 & $>0.05$ \\
\hline & Extension & -0.043 & $>0.05$ \\
\hline \multirow[t]{2}{*}{ Ankle $(n=66)$} & Dorsiflexion & -0.059 & $>0.05$ \\
\hline & Plantar flexion & -0.088 & $>0.05$ \\
\hline
\end{tabular}




\begin{tabular}{|c|c|c|c|c|c|}
\hline Joint & Motion & Group A & Group B & Group C & $p$ value \\
\hline \multirow[t]{5}{*}{ Shoulder } & & $n=10$ & $n=14$ & $n=16$ & \\
\hline & Flexion & $30.0(0.0 ; 60.0)$ & $50.0(0.0 ; 100.0)$ & $40.0(0.0 ; 100.0)$ & $>0.05$ \\
\hline & Abduction & $30.0(0.0 ; 90.0)$ & $50.0(0.0 ; 120.0)$ & $40.0(5.0 ; 130.0)$ & $>0.05$ \\
\hline & Internal rotation & $10.0(0.0 ; 20.0)$ & $15.0(0.0-80.0)$ & $10.0(0.0 ; 60.0)$ & $>0.05$ \\
\hline & External rotation & $20.0(0.0 ; 30.0)$ & $15.0(0.0-80.0)$ & $10.0(0.0 ; 60.0)$ & $>0.05$ \\
\hline \multirow[t]{5}{*}{ Elbow } & & $n=15$ & $n=25$ & $n=18$ & \\
\hline & Flexion & $10.0(0.0 ; 10.0)$ & $10.0(0.0,55.0)$ & $20.0(5.0 ; 60.0)$ & $>0.05$ \\
\hline & Extension & $5.0(0.0 ; 30.0)$ & $10.0(-10.0 ; 60.0)$ & $10.0(-10.0 ; 40.0)$ & $>0.05$ \\
\hline & Supination & $5.0(0.0 ; 10.0)$ & $10.0(-90.0 ; 60.0)$ & $10.0(0.0 ; 50.0)$ & $>0.05$ \\
\hline & Pronation & $5.0(0.0 ; 15.0)$ & $5.0(-90.0 ; 80.0)$ & $10.0(0.0 ; 30.0)$ & $>0.05$ \\
\hline \multirow[t]{3}{*}{ Wrist } & & $n=11$ & $n=23$ & $n=15$ & \\
\hline & Extension & $0.0(0.0 ; 5.0)$ & $20.0(0.0 ; 90.0)$ & $20.0(0.0 ; 90.0)$ & $>0.05$ \\
\hline & Flexion & $0.0(0.0 ; 0.0)$ & $20.0(-5.0 ; 75.0)$ & $27.5(0.0 ; 65.0)$ & $>0.05$ \\
\hline \multirow[t]{6}{*}{ Hip } & & $n=6$ & $n=7$ & $n=5$ & \\
\hline & Flexion & $37.5(10.0 ; 65.0)$ & $20.0(0.0 ; 60.0)$ & $20.0(5.0 ; 30.0)$ & $>0.05$ \\
\hline & Abduction & $5.0(0.0 ; 10.0)$ & $5.0(0.0 ; 10.0)$ & $5.0(0.0 ; 10.0)$ & $>0.05$ \\
\hline & Internal rotation & $-10.0(-20.0 ; 0.0)$ & $5.0(0.0 ; 10.0)$ & $0.0(-30.0 ; 20.0)$ & $>0.05$ \\
\hline & External rotation & $-12.5(-25.0 ; 0.0)$ & $10.0(0.0 ; 10.0)$ & $0.0(0.0 ; 10.0)$ & $>0.05$ \\
\hline & Extension & $5.0(5.0 ; 5.0)$ & $5.0(5.0 ; 5.0)$ & $2.5(0.0 ; 5.0)$ & $>0.05$ \\
\hline \multirow[t]{3}{*}{ Knee } & & $n=47$ & $\mathrm{n}=44$ & $n=39$ & \\
\hline & Flexion & $20.0(5.0 ; 35.0)$ & $30.0(-125.0 ; 115.0)$ & $35.0(-20.0 ; 110.0)$ & $>0.05$ \\
\hline & Extension & $5.0(0.0 ; 10.0)$ & $0.0(-130.0 ; 25.0)$ & $0.0(0.0 ; 20.0)$ & $>0.05$ \\
\hline \multirow[t]{3}{*}{ Ankle } & & $\mathrm{n}=\mathbf{2 0}$ & $\mathrm{n}=25$ & $n=21$ & \\
\hline & Dorsiflexion & $10.0(-5.0 ; 10.0)$ & $7.5(-20.0 ; 40.0)$ & $2.5(-15.0 ; 10.0)$ & $>0.05$ \\
\hline & Plantar flexion & $10.0(5.0-10.0)$ & $10.0(-25.0 ; 40.0)$ & $10.0(0.0 ; 40.0)$ & $>0.05$ \\
\hline
\end{tabular}

\section{Ethics}

Ethics Committee Approval: Approval of the Local Research Ethics Committee of our tertiary hospital was obtained before initiating the study (Gülhane Training and Research Hospital, January 02, 2013, 12 th session).

Informed Consent: The research protocol complies with the Declaration of Helsinki and written informed consent was obtained from all participants.

Peer-review: Externally peer-reviewed.

\section{Authorship Contributions}

Surgical and Medical Practices: D.T., E.Y., E.A., S.K., Ö.K., M.A.T., A.Ö., A.K.T., Concept: D.T., E.Y., E.A., S.K., Ö.K., M.A.T., A.Ö., A.K.T., Design: D.T., E.Y., E.A., S.K., Ö.K., M.A.T., A.Ö., A.K.T., Data Collection or Processing: D.T., E.Y., E.A., S.K., Ö.K., M.A.T., A.Ö., A.K.T., Analysis or Interpretation: D.T., E.Y., E.A., S.K., Ö.K., M.A.T., A.Ö., A.K.T., Literature Search: D.T.,
E.Y., E.A., S.K., Ö.K., M.A.T., A.Ö., A.K.T., Writing: D.T., E.Y., E.A., S.K., Ö.K., M.A.T., A.Ö., A.K.T.

Conflict of Interest: No conflict of interest was declared by the authors.

Financial Disclosure: The authors declared that this study received no financial support.

\section{References}

1. Michlovitz SL, Harris BA, Watkins MP. Therapy interventions for improving joint range of motion, a systematic review. J Hand Ther. 2004;17:118-131.

2. Monument MJ, Hart DA, Salo PT, Befus AD, Hildebrand KA. Posttraumatic elbow contractures: targeting neuroinflammatory fibrogenic mechanisms. J Orthop Sci. 2013;18:869-877.

3. Müller M, Fischer U, Bartoszek G, Grill E, Meyer G. Impact of joint contractures on functioning and social participation in older individuals -- development of a standard set 
(JointConFunctionSet): study protocol. BMC Geriatr. 2013;13:18.

4. Farmer SE, Woollam PJ, Patrick JH, Roberts AP, Bromwich W. Dynamic orthoses in the management of joint contracture. J Bone Joint Surg Br. 2005;87:291-295.

5. Katalinic OM, Harvey LA, Herbert RD, Moseley AM, Lannin NA, Schurr K. Stretch for the treatment and prevention of contractures. Cochrane Database Syst Rev. 2010;9:CD007455.

6. Furia JP, Willis FB, Shanmugam R, Curran SA. Systematic review of contracture reduction in the lower extremity with dynamic splinting. Adv Ther. 2013;30:763-770.

7. Harvey L, Herbert R, Crosbie J. Does stretching induce lasting increases in joint ROM? A systematic review. Physiother Res Int. 2002;7:1-13.

8. Nesterenko S, Morrey ME, Abdel MP, An KN, Steinmann SP, Morrey BF, Sanchez-Sotelo J. New rabbit Knee model of posttraumatic joint contracture: Indirect capsular damage induces a severe contracture. J Orthop Res. 2009;27:10281032.

9. Hildebrand KA. Posttraumatic elbow joint contractures: Defining pathologic capsular mechanisms and potential future treatment paradigsm. J Hand Surg. 2013;38:22272233.

10. Jandric S. Effects of physical therapy in the treatment of the posttraumatic elbow contractures in the children. Bosn J Basic Med Sci. 2007;7:29-32.

11. Lai JM, Francisco GE, Willis FB. Dynamic splinting after treatment with botulinum toxin type-A: a randomized controlled pilot study. Adv Ther. 2009;26:241-248.

12. Berner $\mathrm{SH}$, Willis FB, Martinez J. Treatment of carpal tunnel syndrome with dynasplint: a randomized, controlled trial. J Med. 2008;1:90-94.

13. Gaspar PD, Willis FB. Adhesive capsulitis and dynamic splinting: a controlled, cohort study. BMC Musculoskelet Disord. 2009;10:111-115.

14. Doucet BM, Mettler JA. Effects of a dynamic progressive orthotic intervention for chronic hemiplegia: a case series. J Hand Ther. 2013;26:139-147.
15. Schwartz DA. Static progressive orthoses for the upper extremity: a comprehensive literature review. Hand (N Y). 2012;7:10-17.

16. McGrath MS, Ulrich SD, Bonutti PM, Marker DR, Johanssen $\mathrm{HR}$, Month MA. Static progressive splinting for restoration of rotational motion of the forearm. J Hand Ther. 2009;22:38.

17. Bonutti PM, Windau JE, Ables BA, Miller BG. Static progressive stretch to reestablish elbow range of motion. Clin Orthop Relat Res. 1994;303:128-134.

18. Ulrich SD, Bonutti PM, Seyler TM, Marker DR, Morrey BF, Mont MA. Restoring range of motion via stress relaxation and static progressive stretch in posttraumatic elbow contractures. J Should Elb Surg. 2010;19:196-201.

19. Bhat AK, Bhaskaranand K, Nair SG. Static progressive stretching using a turnbuckle orthosis for elbow stiffness: a prospective study. J Orthop Surg (Hong Kong). 2010;18:7679.

20. Green D, McCoy H. Turnbuckle orthotic correction of elbow contractures after acute injuries. J Bone Joint Surg Am. 1979;61:1092-1095.

21. Lindenhovius AL, Doornberg JN, Brouwer KM, Jupiter JB, Mudgal CS, Ring D. A prospective randomized controlled trial of dynamic versus static progressive elbow splinting for posttraumatic elbow stiffness. J Bone Joint Surg Am. 2012;94:694-700.

22. Harvey LA, Katalinic OM, Herbert RD, Moseley AM, Lannin NA, Schurr K. Stretch for the treatment and prevention of contractures. Cochrane Database Syst Rev. 2017;1:CD007455.

23. Harvey LA, Katalinic OM, Herbert RD, Moseley AM, Lannin NA, Schurr K. Stretch for the treatment and prevention of contracture: an abridged republication of a Cochrane Systematic Review. J Physiother. 2017;63:67-75.

24. Aronson P, Rijke A, Hertel J, Ingersoll CD. Medial tibiofemoral-joint stiffness in males and females across the lifespan. J Athl Train. 2014;49:399-405.

25. Wong K, Trudel G, Laneuville O. Non-Inflammatory Joint Contractures Arising from Immobility: Animal Models to Future Treatments. Biomed Res Int. 2015:2015;848290. 